Pacific Journal of Mathematic 


\title{
TRANSLATION KERNELS ON DISCRETE ABELIAN GROUPS
}

\author{
William R. EMERSon
}

Let $G$ be a compact Abelian group with discrete countable dual group $\Gamma=\hat{G}$ and let $f \in L^{1}(G)$ with Fourier transform $F=\hat{f}$. If $V$ is a finite subset of $\Gamma$ we consider the operator $F_{V}$ on $L^{2}(V)$ :

$$
\left(F_{V} \varphi\right)(\gamma)=\sum_{\tau \in V} F(\gamma-\tau) \varphi(\tau) \quad \varphi \in L^{2}(V), \gamma \in V .
$$

Then if $\left\{V_{n}\right\}$ is any suitably restricted sequence of finite subsets of $\Gamma$ we show that

$$
\lim _{n \rightarrow \infty}\left|F_{V_{n}}\right|=\lim _{n \rightarrow \infty}\left\{\max _{\|\varphi\|_{2}=1}\left|\left(F_{V_{n}} \varphi, \varphi\right)\right|\right\}=\|f\|_{\infty}
$$

where $\left|F_{V}\right|$ is the operator norm of $F_{V}$ on $L^{2}(V)$ and $\left(F_{V} \varphi, \varphi\right)$ denotes the inner product of $F_{V} \varphi$ and $\varphi$ (over $V$ ).

This result is then translated into a statement concerning a special class of infinite matrices which generalize the classical Toeplitz matrices. We then apply these results in evaluating the norm of a special type of linear operator.

In [1] the author considered the asymptotic distribution of eigenvalues and characteristic numbers of certain sequences of operators $\left\{F_{V_{n}}\right\}$ over a locally compact group $\Gamma$ associated with sequences $\left\{V_{n}\right\}$ of Borel sets of $\Gamma$ of finite nonzero measure satisfying

$$
\lim _{n \rightarrow \infty}\left|\gamma V_{n} \triangle V_{n}\right| /\left|V_{n}\right|=0 \quad \text { for all } \gamma \in \Gamma,
$$

where $/ \mid$ is left Haar measure on $\Gamma$. We write $\left\{V_{n}\right\} \in W_{\Gamma}$, and say $\left\{V_{n}\right\}$ has the weak ratio property in case $(*)$ is satisfied (see [2]). In this paper we are considering countable Abelian $\Gamma$ and a more general family $T_{\Gamma}$ of sequences $\left\{V_{n}\right\}$ than those in $W_{\Gamma}$ (and hence in general the asymptotic distribution of the characteristic numbers of $\left\{F_{V_{n}}\right\}$ does not exist, [2]) but still restricted enough to guarantee an asymptotic formula for the maximal characteristic number of $F_{V_{n}}$ as $n \rightarrow \infty$.

1. The basic theorem. $\Gamma$ denotes an arbitrary countably infinite discrete Abelian group equipped with the counting measure.

Definition 1. A sequence $\left\{V_{n}\right\}$ of finite nonempty subsets of $\Gamma$ has the translation property, written $\left\{V_{n}\right\} \in T_{\Gamma}$, if and only if to every finite subset $\Gamma_{0} \leqq \Gamma$ there corresponds an $n_{0}=n_{0}\left(\Gamma_{0}\right)$ such that for $n \geqq n_{0}$ there exists a $\tau_{n}=\tau_{n}\left(\Gamma_{0}\right) \in \Gamma$ with the property that $\tau_{n}+\Gamma_{0} \subseteq V_{n}$. 
Proposition 1. (i) $\left\{V_{n}\right\} \in T_{\Gamma}$ and $V_{n} \subseteq V_{n}^{*}$ for $n \in N^{+}$implies $\left\{V_{n}^{*}\right\} \in T_{\Gamma}$. (ii) $W_{\Gamma}$ is properly contained in $T_{\Gamma}$.

Proof. (i) is immediate from the definition. To prove (ii), first assume $\left\{V_{n}\right\} \in W_{\Gamma}$ and fix any finite nonempty subset $\Gamma_{0} \subseteq \Gamma$. Set $C=\Gamma_{0} \cup\{0\}$. We then readily conclude (see [2] where many properties of $W_{\Gamma}$ are established)

$$
\lim _{n \rightarrow \infty} \frac{\left|C+V_{n}\right|}{\left|V_{n}\right|}=1 \text {. }
$$

But $C+V_{n}=\bigcup_{\tau \in V_{n}}\left(\tau+\Gamma_{0}\right) \cup V_{n}$. Hence if $\tau+\Gamma_{0} \nsubseteq V_{n}$ for all $\tau \in V_{n}$, we have $\left|\left(\tau+\Gamma_{0}\right) \sim V_{n}\right| \geqq 1$ for all $\tau \in V_{n}$ and consequently

$$
\left|C+V_{n}\right| \geqq\left|V_{n}\right|+\frac{\left|V_{n}\right|}{\left|\Gamma_{0}\right|}
$$

since we may choose $\left|V_{n}\right|$ elements $\tau+\gamma_{\tau} \in\left(\tau+\Gamma_{0}\right) \sim V_{n}$, where $\tau \in V_{n}$ and $\gamma_{\tau} \in \Gamma_{0}$, and no element is duplicated more than $\left|\Gamma_{0}\right|$ times. But for sufficiently large $n$ (2) violates (1) and therefore there is a $\tau_{n} \in V_{n}$ for which $\tau_{n}+\Gamma_{0} \subseteq V_{n}$. Hence $W_{\Gamma} \subseteq T_{\Gamma}$.

We now show inclusion is proper. For let $\left\{V_{n}\right\} \in W_{I}(\neq \varnothing$, by [2]); we shall construct a sequence $V_{n}^{*} \supseteqq V_{n}$ such that $\left\{V_{n}^{*}\right\} \notin W_{\Gamma}$, which completes the proof of (ii) upon appealing to (i). Fix any $\gamma \in \Gamma \sim\{0\}$. We inductively construct a sequence $\nu_{1}^{(n)}, \cdots, \nu_{\left|V_{n}\right|}^{(n)}$ as follows: $\nu_{1}^{(n)} \notin V_{n}+\{0, \pm \gamma\}$, and

$$
\nu_{k}^{(n)} \notin\left(V_{n} \cup\left\{\nu_{1}^{(n)}, \cdots, \nu_{k-1}^{(n)}\right\}\right)+\{0, \pm \gamma\} \quad\left(2 \leqq k \leqq\left|V_{n}\right|\right) .
$$

We set $V_{n}^{*}=V_{n} \cup\left\{\nu_{1}^{(n)}, \cdots, \nu_{|V|}^{(n)}\right\}$ and verify that

$$
\frac{\left|\left(\gamma+V_{n}^{*}\right) \cap V_{n}^{*}\right|}{\left|V_{n}^{*}\right|} \leqq \frac{1}{2}
$$

implying $\left\{V_{n}^{*}\right\} \notin W_{\Gamma}$. For

$$
\begin{aligned}
& \left(\gamma+V_{n}^{*}\right) \cap V_{n}^{*} \\
= & \left(\left(\gamma+V_{n}\right) \cap V_{n}^{*}\right) \cup\left(\left\{\gamma+\nu_{1}^{(n)}, \cdots, \gamma+\nu_{\mid V_{n}}^{(n)}\right\} \cap\left(V_{n} \cup\left\{\nu_{1}^{(n)}, \cdots, \nu_{\left.\mid V_{n}\right\}}^{(n)}\right\}\right)\right) \\
= & \left(\left(\gamma+V_{n}\right) \cap V_{n}^{*}\right)
\end{aligned}
$$

since the second term in the union is empty by (I). Hence $\left|\left(\gamma+V_{n}^{*}\right) \cap V_{n}^{k}\right| \leqq\left|\gamma+V_{n}\right|=\left|V_{n}\right|$, and therefore for $n \in N^{+}$

$$
\frac{\left|\left(\gamma+V_{n}^{*}\right) \cap V_{n}^{*}\right|}{\left|V_{n}^{*}\right|}=\frac{\left|\left(\gamma+V_{n}^{*}\right) \cap V_{n}^{*}\right|}{2\left|V_{n}\right|} \leqq \frac{\left|V_{n}\right|}{2\left|V_{n}\right|}=\frac{1}{2} .
$$

We now prove a result, of independent interest, which is critical in the proof of Theorem 1 . 
Proposition 2. Let $G$ be a compact Abelian group (with measure normalized to one), let $f \in L^{1}(G)$, and let $\rho$ be any positive number. Then

$$
\|f\|_{\infty}=\left.\sup _{\omega}\left|\int_{G}\right| \omega(x)\right|^{\rho} f(x) d x \mid
$$

where $\omega$ ranges over all trigonometric polynomials on $G$ satisfying $\|\omega\|_{\rho} \leqq 1$.

Proof. Recall that a trigonometric polynomial is a finite linear combination of characters on $G$. Clearly

$$
\left.\left.\left|\int_{G}\right| \omega(x)\right|^{\rho} f(x) d x\left|\leqq\|f\|_{\infty} \int_{G}\right| \omega(x)\right|^{\rho} d x=\|f\|_{\infty}\|\omega\|_{\rho}^{\rho} \leqq\|f\|_{\infty} .
$$

We divide the proof of the converse inequality into two cases:

To prove the converse inequality, we first consider the case $\|f\|_{\infty}<+\infty$. Fix any $\delta>0$ (until the conclusion of the argument). Let $S=S(\delta)$ be a measurable subset of the complex plane of diameter less than $\delta$ and such that

$$
E=f^{-1}(S),\left\|\chi_{E} f\right\|_{\infty}=\|f\|_{\infty},
$$

where $\chi_{E}$ denotes the characteristic function of $E$. Hence for $s \in S$ and $x \in E$ we have

$$
|| s|-|\left(\chi_{E} f\right)(x)|| \leqq\left|s-\left(\chi_{E} f\right)(x)\right|<\delta,
$$

and consequently also

$$
|| s\left|-\|f\|_{\infty}\right|=|| s\left|-\left\|\chi_{E} f\right\|_{\infty}\right| \leqq \delta .
$$

Therefore, if $g=\chi_{E} /|E|$ then

$$
\begin{aligned}
0 & \leqq\|f\|_{\infty}-\left|\int_{G} f g d x\right| \leqq|| f \|_{\infty}-|s||+||s|-\left|\int_{G} f g d x\right| \mid \\
& \leqq\left|\|f\|_{\infty}-\right| s||+\left|s-\int_{G} f g d x\right| \\
& =\left|\|f\|_{\infty}-\right| s||+\left|\frac{1}{|E|} \int_{E}\left(s-\chi_{E} f\right) d x\right| \leqq 2 \delta .
\end{aligned}
$$

We next wish to approximate $g$ by a continuous function $h$, and at this point the estimate is rather delicate because this is also needed later in the case $\|f\|_{\infty}=+\infty$ and consequently we must avoid $\|f\|_{\infty}$ as a factor in the error of estimation. Now since $f \in L^{1}(G)$, to every $\varepsilon>0$ there corresponds an $\eta=\eta(\varepsilon)$ such that for all measurable subsets $T$ of $G$ of measure at most $\eta$ 


$$
\int_{T}|f| d x<\varepsilon
$$

We now choose $\gamma=\gamma(\delta)$ satisfying

(i) $\gamma<\delta|E|$,

(ii) $\int_{T}|f| d x<\delta|E|$ if $|T|<\gamma$.

Furthermore, since Haar measure is regular, we may find an open set $E^{+}$and a closed set $E^{-}$such that

$$
E^{-} \sqsubseteq E \leqq E^{+}, \quad\left|E^{+} \sim E^{-}\right|<\gamma
$$

Finally (by Urysohn's Lemma, since $G$ is a normal topological space) there exists a continuous $h_{0}: G \rightarrow[0,1]$ such that $h_{0} \mid E^{-} \equiv 1$ and $h_{0} \mid G \sim E^{+} \equiv 0$. Our candidate for $h$ is then defined to be the nonnegative function $h=h_{0} /|E|$. Let us now estimate $\int_{G} f g d x-\int_{G} f h d x$ :

$$
\begin{aligned}
& \left|\int_{G} f g d x-\int_{G} f h d x\right| \leqq \int_{G}|f||g-h| d x \\
= & \left(\int_{E^{-}}+\int_{E^{+} \sim E^{-}}+\int_{G \sim E^{+}}\right)|f||g-h| d x=\int_{E^{+} \sim E^{-}}|f||g-h| d x \\
& \leqq \max |g-h| \int_{E^{+} \sim E^{-}}|f| d x \leqq \frac{1}{|E|} \cdot \delta|E|=\delta
\end{aligned}
$$

by (4), (4') and the definitions of $g$ and $h$. Also, we have

$$
\int_{E^{-}} h d x \leqq \int_{G} h d x=\int_{E^{+}} h d x \leqq\|h\|_{\infty}\left|E^{+}\right|
$$

implying the estimate

$$
\frac{\left|E^{-}\right|}{|E|} \leqq\|h\|_{1} \leqq \frac{\left|E^{+}\right|}{|E|} \leqq 1+\delta \text { by virtue of (4) and }\left(4^{\prime}\right) \text {. }
$$

Lastly, to any $\alpha>0$ we may correspond a trigonometric polynomial $\omega_{\alpha}$ satisfying $\left\|h^{1 / \rho}-\omega_{\alpha}\right\|_{\infty}<\alpha$, and consequently $\left\|h^{1 / \rho}-\left|\omega_{\alpha}\right|\right\|_{\infty}<\alpha$ since $h^{1 / \rho} \geqq 0$. Thus by choosing $\alpha_{0}=\alpha_{0}(\delta)$ sufficiently small we may conclude

$$
\left\|h-\left|\omega_{\alpha_{0}}\right|^{\rho}\right\|_{1} \leqq\left\|h-\left|\omega_{\alpha_{0}}\right|^{\rho}\right\|_{\infty}<\delta
$$

Also,

$$
\left\|\omega_{\alpha_{0}}^{o}\right\|_{1} \leqq\left\|h-\left|\omega_{\alpha_{0}}\right|^{\rho}\right\|_{1}+\|h\|_{1} \leqq \delta+(1+\delta)=1+2 \delta .
$$

We now let

$$
\omega=\omega_{\alpha_{0}} /(1+2 \delta)^{1 / \rho} \text {, implying }\|\omega\|_{\rho} \leqq 1
$$


Finally,

$$
\begin{aligned}
& \left.\left|\int_{G}\right| \omega\right|^{\rho} f d x\left|=\frac{1}{1+2 \delta}\right| \int_{G}\left|\omega_{\alpha_{0}}\right|^{\rho} f d x \mid \\
\geqq & \frac{1}{1+2 \delta}\left(\left|\int_{G} f g d x\right|-\left|\int_{G} f g d x-\int_{G} f h d x\right|-\left.\left|\int_{G} h f d x-\int_{G}\right| \omega_{\alpha_{0}}\right|^{\rho} f d x \mid\right) \\
\geqq & \frac{1}{1+2 \delta}\left(\left(\|f\|_{\infty}-2 \delta\right)-\delta-\delta\|f\|_{1}\right) .
\end{aligned}
$$

By (3), (5), and (7). Our assertion follows upon letting $\delta \rightarrow 0$.

In case $\|f\|_{\infty}=+\infty$, we let $S_{n}$ be a measurable subset of the complex plane of diameter less than $\delta$ and such that $E_{n}=f^{-1}\left(S_{n}\right)$, $\left\|\chi_{E_{n}} f\right\|_{\infty}>n$. Equations (3) - (8) still hold with $\|f\|_{\infty}$ replaced by $\left\|\chi_{E_{n}} f\right\|_{\infty}>n$ wherever it occurs, and we readily construct trigonometric polynomials $\omega_{n}$ with $\left\|\omega_{n}\right\|_{\rho} \leqq 1$ and such that $\int_{G}\left|\omega_{n}\right|^{\rho} f d x$ is unbounded as $n \rightarrow+\infty$.

We now are ready to prove the basic theorem.

THEOREM 1. Let $G$ be a compact group (with measure normalized to one), let $f \in L^{1}(G)$, and let $F=\hat{f} \in L^{\infty}(\Gamma)$, the Fourier Transform of $f$. Furthermore, let $\left\{V_{n}\right\} \in T_{\Gamma}$ and let $F_{V_{n}}$ be the Hilbert-Schmidt operator on $L^{2}\left(V_{n}\right)$ :

$$
\left(F_{V_{n}} \psi\right)(\gamma)=\int_{V_{n}} F(\gamma-\tau) \psi(\tau) d \tau=\sum_{\tau \in V_{n}} F(\gamma-\tau) \psi(\tau)
$$

$$
\left(\psi \in L^{2}\left(V_{n}\right), \gamma \in V_{n}\right) \text {. }
$$

Let $\left(F_{V_{n}} \psi, \psi\right)_{V_{n}}$ denote the inner product of $F_{V_{n}} \psi$ and $\psi$ over $V_{n}$, and let $\left|F_{V_{n}}\right|$ denote the maximal characteristic number of $F_{V_{n}}$ as an operator on the Hilbert space $L^{2}\left(V_{n}\right)$. Then

$$
\begin{aligned}
& \lim _{n \rightarrow \infty} \max _{\|\psi\|_{2}=1}\left|\left(F_{V_{n}} \psi, \psi\right)_{V_{n}}\right|=\|f\|_{\infty} . \\
& \lim _{n \rightarrow \infty}\left|F_{V_{n}}\right|=\|f\|_{\infty} .
\end{aligned}
$$

Proof. (i) By definition,

$$
\begin{aligned}
& \left(F_{V_{n}} \psi, \psi\right)_{V_{n}}=\sum_{\gamma, \tau \in V_{n}} F(\gamma-\tau) \psi(\tau) \psi \overline{(\gamma)} \\
= & \sum_{\gamma, \tau \in V_{n}}\left[\int_{G} \overline{(\gamma-\tau, x)} f(x) d x\right] \psi(\tau) \overline{\psi(\gamma)} \\
= & \int_{G}\left[\sum_{r, \tau \in V_{n}}(\tau, x) \psi(\tau) \overline{(\gamma, x) \psi(\gamma)}\right] f(x) d x \\
= & \int_{G}\left|\sum_{\tau \in V_{n}}(\tau, x) \psi(\tau)\right|^{2} f(x) d x .
\end{aligned}
$$

Note that 


$$
\omega_{\psi}(x)=\sum_{\tau \in V_{n}}(\tau, x) \psi(\tau)
$$

is a trigonometric polynomial on $G$, and $\psi \rightarrow \omega_{\psi}$ is an isometry of $L^{2}\left(V_{n}\right)$ into $L^{2}(G)$ since $\left\|\omega_{\psi}\right\|_{2}^{2}=\sum_{\tau \in V_{n}}|\psi(\tau)|^{2}=\|\psi\|_{2}^{2}$. Therefore

$$
\max _{\|\psi\|_{2}=1}\left|\left(F_{V_{n}} \psi, \psi\right)_{V_{n}}\right|=\left.\max _{\| \omega !_{2}=1}\left|\int_{G}\right| \omega(x)\right|^{2} f(x) d x
$$

where $\omega$ ranges over linear combinations of characters on $G$ generated by elements in $V_{n}$. Hence by Proposition $2(\rho=2)$,

$$
\lim _{n \rightarrow \infty} \max _{\left\|\psi_{\gamma}\right\|_{2}=1}\left|\left(F_{V_{n}} \psi, \psi\right)_{V_{n}}\right| \leqq\|f\|_{\infty}
$$

On the other hand, let $\omega$ be any trigonometric polynomial on $G$, say

$$
\omega(x)=\sum_{1 \leqq i \leqq k}\left(\gamma_{i}, x\right) c_{i} \quad\left(c_{i} \in \mathscr{C}, \gamma_{i} \in \Gamma\right) .
$$

Let $\Gamma_{0}=\left\{\gamma_{1}, \cdots, \gamma_{k}\right\}$, a finite subset of $\Gamma$. Now since $\left\{V_{n}\right\} \in T_{\Gamma}$ there exists an $n_{0}$ such that for $n \geqq n_{0}$ there exists $\tau_{n} \in \Gamma$ such that $\tau_{n}+\Gamma_{0} \subseteq V_{n}$. Hence for $n \geqq n_{0}$,

$$
\omega_{n}(x)=\left(\tau_{n}, x\right) \omega(x)=\sum_{1 \leqq i \leqq k}\left(\tau_{n}+\gamma_{i}, x\right) c_{i}
$$

is a linear combination of characters on $G$ generated by elements of $V_{n}$. Since $|\omega(x)|=\left|\omega_{n}(x)\right|$ for all $x \in G$, the proof of (i) is completed by again applying Proposition 2 with $\rho=2$.

(ii) Recall that $\left|F_{V_{n}}\right|$ is the norm of $F_{V_{n}}$ considered as an operator on $L^{2}\left(V_{n}\right)$, i.e.,

$$
\left|F_{V_{n}}\right|=\max _{\|\psi\|_{2}=1}\left\|F_{V_{n}} \psi\right\|_{2} \text {. }
$$

but by the Cauchy-Schwarz Inequality, for $\|\psi\|_{2}=1$

$$
\left|\left(F_{V_{n}} \psi, \psi\right)_{V_{n}}\right| \leqq\left\|F_{V_{n}} \psi\right\|_{2}\|\psi\|_{2}=\left\|F_{V_{n}} \psi\right\|_{2} \leqq\left|F_{V_{n}}\right|
$$

and therefore by (i),

$$
\lim _{n \rightarrow \infty}\left|F_{V_{n}}\right| \geqq \lim _{n \rightarrow \infty} \max _{\|\psi\|_{2}=1}\left|\left(F_{V_{n}} \psi, \psi\right)_{V_{n}}\right|=\|f\|_{\infty}
$$

Thus, if $\|f\|_{\infty}=+\infty$ nothing remains to be proved. If $\|f\|_{\infty}<+\infty$ we have $f \in L^{1}(G) \cap L^{\infty}(G)$, and therefore by [3], p. 445, $\left|F_{V_{n}}\right| \leqq\|f\|_{\infty}$ for all $n \in N^{+}$. Hence $\overline{\lim }_{n \rightarrow \infty}\left|F_{V_{n}}\right| \leqq\|f\|_{\infty}$, and consequently $\lim _{n \rightarrow \infty}\left|F_{V_{n}}\right|=\|f\|_{\infty}$ in this case as well. 
We now conversely prove that the hypothesis $\left\{V_{n}\right\} \in T_{\Gamma}$ is in fact necessary for the conclusion of Theorem 1. More precisely,

THEOREM 1'. Using the notation of Theorem 1 , if $\left\{V_{n}\right\}$ is any sequence of finite subsets of $\Gamma$ for which conclusion (i) holds for all trigonometric polynomials $f$ on $G$, then $\left\{V_{n}\right\} \in T_{\Gamma}$.

Proof. Assume $\left\{V_{n}\right\} \notin T_{\Gamma}$, i.e., there exists a finite subset $\Gamma_{0}$ of $\Gamma$ such that no translate of $\Gamma_{0}$ lies in $V_{m}$ for an appropriate subsequence $m \rightarrow \infty$. We then assert that

$$
f(x)=\frac{1}{\left|\Gamma_{0}\right|} \sum_{\tau \in \Gamma_{0}}(\tau, x) \quad\left(\|f\|_{\infty}=f(0)=1\right)
$$

is a trigonometric polynomial for which (i) fails. More precisely we show for all these $m$ :

$$
\operatorname{Max}_{\|\psi\|_{2}=1}\left|\left(F_{V_{m}} \psi, \psi\right)_{V m}\right| \leqq\left(1-\frac{1}{2\left|\Gamma_{0}\right|}\right)\|f\|_{\infty}
$$

Recalling relation ( $\dagger$ ) of the proof of Theorem 1 . We have:

$$
\operatorname{Max}_{\|\psi\|_{2}=1}\left|\left(F_{V m} \psi, \psi\right)_{V m}\right|=\left.\operatorname{Max}_{\|\omega\|_{2}=1}\left|\int_{G}\right| \omega(x)\right|^{2} f(x) d x \mid
$$

where $\omega$ ranges over all linear combinations of characters on $G$ generated by elements in $V_{m}$.

However, any such $\omega$ is of the form

$$
\omega(x)=\sum_{\tau \in V m}(\tau, x) a_{\tau}
$$

where

$$
\sum_{\tau \in V_{m}}\left|a_{\tau}\right|^{2}=\|\omega\|_{2}^{2} \leqq 1
$$

implying

$$
|\omega(x)|^{2}=\sum_{\tau_{1}, \tau_{2} \in V_{m}}\left(\tau_{1}-\tau_{2}, x\right) a_{\tau_{1}} \bar{a}_{\tau_{2}}
$$

and finally

$$
\int_{G}|\omega(x)|^{2} f(x) d x=\frac{1}{\left|\Gamma_{0}\right|} \sum_{\substack{\tau_{1}, \tau_{2} \in V_{m} \\ \tau_{2}-\tau_{1} \in T_{0}}} a_{\tau_{1}} \bar{a}_{\tau_{2}} .
$$

Consequently, 


$$
\begin{aligned}
\left.\left|\int_{G}\right| \omega(x)\right|^{2} f(x) d x \mid \leqq & \frac{1}{\left|\Gamma_{0}\right|} \sum_{\substack{\tau_{1}, \tau_{2} \in V_{m} \\
\tau_{2}-\tau_{1} \in \Gamma_{0}}}\left|a_{\tau_{1}}\right|\left|a_{\tau_{2}}\right| \leqq \frac{1}{2\left|\Gamma_{0}\right|} \sum_{\substack{\tau_{1}, \tau_{2} \in V_{m} \\
\tau_{2}-\tau_{1} \in T_{0}}}\left(\left|a_{\tau_{1}}\right|^{2}+\left|a_{\tau_{2}}\right|^{2}\right) \\
= & \frac{1}{2\left|\Gamma_{0}\right|}\left(\sum_{\tau_{1} \in V_{m}}\left|a_{\tau_{1}}\right|^{2}\left(\sum_{\substack{\tau_{2} \in V_{m} \\
\tau_{2}-\tau_{1} \in \Gamma_{0}}} 1\right)+\sum_{\tau_{2} \in V_{m}}\left|a_{\tau_{2}}\right|^{2}\left(\sum_{\substack{\tau_{1} \in V_{m} \\
\tau_{2}-\tau_{1} \in \Gamma_{0}}} 1\right)\right) \\
= & \frac{1}{2\left|\Gamma_{0}\right|}\left(\sum_{\tau_{1} \in V_{m}}\left|a_{\tau_{1}}\right|^{2}\left|\left(V_{m}-\tau_{1}\right) \cap \Gamma_{0}\right|\right. \\
& \left.+\sum_{\tau_{2} \in V_{m}}\left|a_{\tau_{2}}\right|^{2}\left|\left(\tau_{2}-V_{m}\right) \cap \Gamma_{0}\right|\right) \\
\leqq & \frac{1}{2\left|\Gamma_{0}\right|}\left\{\left(\left|\Gamma_{0}\right|-1\right) \sum_{\tau_{1} \in V_{m}}\left|a_{\tau_{1}}\right|^{2}+\left|\Gamma_{0}\right| \sum_{\tau_{2} \in V_{m}}\left|a_{\tau_{2}}\right|^{2}\right\} \\
= & \left(1-\frac{1}{2\left|\Gamma_{0}\right|}\right) \sum_{\tau \in V_{m}}\left|a_{\tau}\right|^{2} \leqq\left(1-\frac{1}{2\left|\Gamma_{0}\right|}\right) \\
= & \left(1-\frac{1}{2\left|\Gamma_{0}\right|}\right)\|f\|_{\infty}
\end{aligned}
$$

since no translate $V_{m}-\tau_{1}$ contains $\Gamma_{0}$ by hypothesis. Our assertion now readily follows.

2. A class of doubly-infinite matrices. We now translate the theorem of the preceding section into a statement concerning a class of doubly-infinite complex matrices $M=\left(\alpha_{i, j}\right)_{i, j=1}^{\infty}$ whose entries $\alpha_{i, j}$ are determined by a "group law".

Definition 2. Let $M=\left(\alpha_{i, j}\right)_{i, j=1}^{\infty}$ be a matrix with complex entries. We then write

$$
M \sim(\Gamma, \Lambda, F)
$$

if and only if

(i) $\Gamma$ is a countable Abelian group.

(ii) $\Lambda$ is a subset of $\Gamma$.

(iii) $F: \Gamma \rightarrow \mathscr{C}$.

(iv) There exists an ordering of $A=\left\{\lambda_{1}, \cdots, \lambda_{n}, \cdots\right\}$ such that for all $i, j \in N^{+}$,

$$
\alpha_{i, j}=F\left(\lambda_{i}-\lambda_{j}\right)
$$

REMARK. For any $M=\left(\alpha_{i, j}\right)_{i, j=1}^{\infty}$ with complex entries we may take $\Gamma$ to be $Q^{\times}$, the multiplicative group of rational numbers, and $\Lambda$ to be $P=\left\{p_{n}: n \in N^{+}\right\}$, the set of all positive integral primes, upon defining $F$ by

$$
F(r)= \begin{cases}\alpha_{i, j} & \text { if } r=p_{i} / p_{j} \\ 0 & \text { otherwise }\end{cases}
$$


We then have $M \sim\left(Q^{\times}, P, F\right)$.

Under suitable restrictions on $(\Gamma, \Lambda, F)$ we shall be able to compute the norm and quadratic norm of the matrix $M$, which are defined as follows:

DEFINITION 3. The norm of $M,|M|$, and the quadratic norm of $M,|M|_{I}$, are defined by

$$
|M|=\sup _{\|X\|_{2} \leq 1}\|M X\|_{2}, \quad|M|_{I}=\sup _{\|X\|_{2} \leqq 1}|(M X, X)|,
$$

where $X=\left(\left\{x_{i}\right\}\right)$ ranges over elements of the complex Hilbert space $l^{2}$ with only finitely many $x_{i} \neq 0$, and $M X=\left(\left\{\sum_{j} \alpha_{i, j} x_{j}\right\}\right)$.

LEMmA 1. If $M$ induces a bounded operator on $l^{2}$, then

(i) $|M|=\sup _{\|X\|_{2} \leqq 1}\|M X\|, \quad$ (ii) $|M|_{I}=\sup _{\|X\|_{2} \leqq 1}|(M X, X)|$,

where $X=\left(\left\{x_{i}\right\}\right)$ ranges over all elements of $l^{2}$ (with $\|X\|_{2} \leqq 1$ ). Hence in this case $|M|$ is the standard norm of $M$ considered as a bounded linear operator on $l^{2}$.

Proof. For $x \in l^{2}$, let $X_{n}$ be the projection of $X$ on its first $n$ components ( 0 elsewhere). Since $M$ is bounded and consequently closed, $\lim _{n \rightarrow \infty} M X_{n}=M X$ and (i) follows since $X_{n}$ has at most $n$ nonzero components. Also

$$
(M X, X)=\left(M X_{n}, X_{n}\right)+\left(M\left(X-X_{n}\right), X_{n}\right)+\left(M X, X-X_{n}\right),
$$

and therefore

$$
\begin{aligned}
& \left|(M X, X)-\left(M X_{n}, X_{n}\right)\right| \\
\leqq & \left\|M\left(X-X_{n}\right)\right\|_{2}\left\|X_{n}\right\|_{2}+\|M X\|_{2}\left\|X-X_{n}\right\|_{2} \rightarrow 0
\end{aligned}
$$

as $n \rightarrow \infty$, and (ii) clearly follows.

THEOREM 2. Let $M \sim(\Gamma, \Lambda, F)$ where

(i) $F \in A(\Gamma)$, i.e., $F=\widehat{f}$ for some $f \in L^{1}(G)$.

(ii) To each finite subset $\Gamma_{0} \subseteq \Gamma$ there corresponds $a \gamma=\gamma\left(\Gamma_{0}\right)$ such that $\gamma+\Gamma_{0} \subseteq \Lambda$.

Then $|M|=|M|_{I}=\|f\|_{\infty}$.

Proof. Assume $\Lambda=\left\{\lambda_{1}, \cdots, \lambda_{n}, \cdots\right\}$ as in Definition 2, and set $V_{n}=\left\{\lambda_{1}, \cdots, \lambda_{n}\right\}$. Then hypothesis (ii) clearly implies $\left\{V_{n}\right\} \in T_{\Gamma}$. The theorem will follow from the two inequalities

(i) $|M| \leqq\|f\|_{\infty}$

(ii) $\|f\|_{\infty} \leqq|M|_{I}$, 
since $|M|_{I} \leqq|M|$ by the Cauchy-Schwarz inequality.

(i): If $\|f\|_{\infty}=+\infty$ there is nothing to prove. Otherwise $f \in L^{1}(G) \cap L^{\infty}(G)$, and therefore the operator $M^{\prime}: L^{2}(\Gamma) \rightarrow L^{2}(\Gamma)$ defined by

$$
\left(M^{\prime} \varphi\right)(\gamma)=\sum_{\tau \in \Gamma} F(\gamma-\tau) \varphi(\tau) \quad\left(\varphi \in L^{2}(\Gamma), \gamma \in \Gamma\right)
$$

has norm $\left|M^{\prime}\right|=\|f\|_{\infty}$ by [3], $\S 3.2 .$, p. 441. Hence if we only consider $\phi$ with support in $\Lambda$ and restrict $\gamma$ to $\Lambda, M^{\prime}$ restricts to an operator $M^{\prime \prime}: L^{2}(\Lambda) \rightarrow L^{2}(\Lambda)$ with $\left|M^{\prime \prime}\right| \leqq\left|M^{\prime}\right|$. Now consider the isometry of $l^{2}$ onto $L^{2}(\Lambda)$ given by $X=\left(\left\{x_{n}\right\}\right) m \varphi_{X}$ where $\varphi\left(\lambda_{n}\right)=x_{n}$ for $n \in N^{+}$. Then for this $\varphi=\varphi_{X}$ and $\lambda_{i} \in \Lambda$,

$$
\left(M^{\prime \prime} \varphi\right)\left(\lambda_{i}\right)=\sum_{\lambda \in A} F\left(\lambda_{i}-\lambda\right) \varphi(\lambda)=\sum_{j} F\left(\lambda_{i}-\lambda_{j}\right) \varphi\left(\lambda_{j}\right)=\sum_{j} \alpha_{i, j} x_{j}
$$

which is the $i^{\text {th }}$ component of $M X$, and therefore $|M|=\left|M^{\prime \prime}\right| \leqq$ $\left|M^{\prime}\right|=\|f\|_{\infty}$ (and thus $M$ induces a bounded linear operator if $\left.\|f\|_{\infty}<+\infty\right)$.

(ii): For $n \in N^{+}$, consider the isometry of $L^{2}\left(V_{n}\right)$ (which is none other than $n$-dimensional Euclidean space) into $l^{2}$ given by $\varphi \rightarrow X_{\varphi}$ where $X_{\varphi}=\left(\left\{x_{j}^{\varphi}\right\}\right)$ and $x_{j}^{\varphi}=\varphi\left(\lambda_{j}\right)$ for $1 \leqq j \leqq n$ and 0 otherwise. Hence $X_{\varphi}$ has only finitely many nonzero components, and each $X \in l^{2}$ with only finitely many nonzero components is in the image of $L^{2}\left(V_{n}\right)$ under the above isometry for $n=n(X)$ sufficiently large. Now consider $F_{V_{n}}$ on $L^{2}\left(V_{n}\right)$ :

$$
\begin{aligned}
\left(F_{V_{n}} \varphi, \varphi\right)_{V_{n}} & =\sum_{1 \leqq i \leqq n} \sum_{1 \leqq j \leqq n} F\left(\lambda_{i}-\lambda_{j}\right) \varphi\left(\lambda_{j}\right) \varphi \overline{\left(\lambda_{i}\right)} \\
& =\sum_{1 \leqq i, j \leqq n} \alpha_{i, j} x_{j} \bar{x}_{i}=\left(M X_{\varphi}, X_{\varphi}\right)
\end{aligned}
$$

But by Theorem 1 (i) $\lim _{n \rightarrow \infty} \max _{\|\varphi\|_{2}=1}\left|\left(F_{V_{n}} \varphi, \varphi\right)_{V_{n}}\right|=\|f\|_{\infty}$ and therefore $|M|_{I}=\|f\|_{\infty}$ since

$$
|M|_{I}=\sup _{\substack{|| x \mid I_{1} \leq 1 \\ x_{\imath} \neq 0 \text { finitely }}}|(M X, X)|=\lim _{n \rightarrow \infty} \max _{\|\varphi\|_{2} \leq 1}\left|\left(F_{V_{n}} \varphi, \varphi\right)_{V_{n}}\right|=\|f\|_{\infty} .
$$

Corollary 1. (1) Hypothesis (i) of Theorem 2 is satisfied if ( i $)^{\prime}$

$$
\sum_{\gamma \in \Gamma}|F(\gamma)|^{2}<+\infty \text {. }
$$

(2) Hypothesis (ii) of Theorem 2 is satisfied if

$$
\Lambda+\Lambda \subseteq \Lambda \text { and (ii)" } \Lambda \text { generates } \Gamma \text {. }
$$

Proof. (1), (i) implies $f(x)=\sum_{\gamma \in \Gamma}(\gamma, x) F(\gamma) \in L^{2}(G)$ and therefore also $f \in L^{1}(G)$ since $G$ is compact and hence of finite measure. Clearly $F=\widehat{f} \in A(\Gamma)$. 
(2) First note that any element of $\Gamma$ is the difference of two elements in $\Lambda$, i.e., $\Gamma=\Lambda-\Lambda$. For by (ii)", if $\gamma \in \Gamma$ we have $\gamma=\lambda_{i_{1}}+\cdots+\lambda_{i_{k}}-\cdots-\lambda_{i_{n}}$ for some suitable finite sequence of integers $i_{1}, \cdots, i_{n}$ (if no terms with a plus sign occur we may take $k=0$, if none with a minus sign occur take $k=n$ ). But by (ii)', $\gamma^{+}=\lambda_{i_{1}}+\cdots+\lambda_{i_{k}} \in \Lambda$ if $k>0, \gamma^{-}=\lambda_{i_{k+1}}+\cdots+\lambda_{i_{n}} \in \Lambda$ if $k<n$. If $k=0$ we may write $\gamma=\lambda_{1}-\left(\lambda_{1}+\cdots+\lambda_{n}+\lambda_{1}\right)$ and similarly $\gamma=\left(\lambda_{1}+\cdots+\lambda_{n}+\lambda_{1}\right)-\lambda_{1}$ if $k=n$.

Now let $\Gamma_{0}=\left\{\gamma_{1}, \cdots, \lambda_{k}\right\}$ be a nonempty finite subset of $\Gamma$. Then for appropriate $a_{i}, b_{i} \in N^{+}$we have

$$
\gamma_{i}=\lambda_{a_{i}}-\lambda_{b_{i}} \quad(1 \leqq i \leqq k) .
$$

Consequently, for $1 \leqq i \leqq k$,

$$
\gamma_{i}=\lambda_{a_{i}}+\lambda_{b_{1}}+\cdots+\hat{\lambda}_{b_{i}}+\cdots+\lambda_{b_{k}}-\left(\lambda_{b_{1}}+\cdots+\lambda_{b_{k}}\right)
$$

where $\left({ }^{\wedge}\right)$ denotes deletion of a term. Hence if we set $\gamma=$ $\lambda_{b_{1}}+\cdots+\lambda_{b_{k}}$ we have $\gamma+\Gamma_{0} \leqq \Lambda$ since

$$
\lambda_{a_{i}}+\lambda_{b_{1}}+\cdots+\hat{\lambda}_{b_{i}}+\cdots+\lambda_{b_{k}} \in \Lambda
$$

by (ii)'.

We now apply Theorem 2 to completely solve the norm evaluation in the case $M \sim(\Gamma, \Lambda, F)$ where $F \geqq 0$ and $\Lambda$ satisfies (ii). We make use of the following simple lemma:

Lemma 2. If $M=\left(\alpha_{i, j}\right)_{i, j=1}^{\infty}$ and $M^{\prime}=\left(\alpha_{i, j}^{\prime}\right)_{i, j=1}^{\infty}$ where

$$
\alpha_{i, j} \geqq \alpha_{i, j}^{\prime} \geqq 0 \quad \text { for all } i, j \in N^{+}
$$

then

$$
|M|=\sup _{\|x\|_{2} \leqq 1}\|M X\|, \quad|M|_{I}=\sup _{\|x\|_{2} \leqq 1}(M X, X)
$$

where $X=\left(\left\{x_{i}\right\}\right)$ has only finitely many nonzero coordinates, all positive.

$$
\left|M^{\prime}\right| \leqq|M|, \quad\left|M^{\prime}\right|_{I} \leqq|M|_{I}
$$

Proof. For $X=\left(\left\{x_{i}\right\}\right) \in l^{2}$ we define $X^{+}=\left(\left\{\left|x_{i}\right|\right\}\right)$. Note $\|X\|_{2}=$ $\left\|X^{+}\right\|_{2}$ and $X^{+}$and $X$ have the same cardinality of nonzero coordinates. Also, $\alpha_{i, j} \geqq 0$ clearly implies $\|M X\|_{2} \leqq\left\|M X^{+}\right\|_{2}$ and $\left\{(M X, X) \mid \leqq\left(M X^{+}, X^{+}\right)\right.$and (i) readily follows. But $\alpha_{i, j} \geqq \alpha_{i, j}^{\prime} \geqq 0$ also implies each component of $M^{\prime} X^{+}$is dominated by the corresponding component of $M X^{+}$and hence (ii) follows from (i).

THEOREM 3. Let $M \sim(\Gamma, \Lambda, F)$ where 
(i) $F(\gamma) \geqq 0$ for all $\gamma \in \Gamma$.

(ii) If $\Gamma_{0}$ is any finite subset of $\Gamma$ there exists a $\gamma=\gamma\left(\Gamma_{0}\right)$ such that $\gamma+\Gamma_{0} \leqq \Lambda$. Then

$$
|M|=|M|_{I}=\sum_{\gamma \in T} F(\gamma) \quad(\text { possibly }+\infty) .
$$

Proof. Since $|M|_{I} \leqq|M|$ it suffices to show that
(1) $|M| \leqq \sum_{\gamma \in \Gamma} F(\gamma)$ ，
(2) $|M|_{I}=\sum_{\gamma \in \Gamma} F(\gamma)$.

If $\sum_{r \in \Gamma} F(\gamma)<+\infty$ there is nothing to prove since in this case the result is included in Theorem 2 because $f(x)=\sum_{\gamma \in \Gamma}(\gamma, x) F(\gamma)$ is a continuous function on $G, F=\hat{f}$, and $\|f\|_{\infty}=f(0)=\sum_{\gamma \in \Gamma} F(\gamma)$.

On the other hand, if $\sum_{\gamma \in \Gamma} F(\gamma)=+\infty$ then $F$ may not be in $A(\Gamma)$ and hence we cannot apply Theorem 2 directly. Clearly (1) is true in this case and we need only verify (2). Let $\Gamma^{\prime}=\left\{\gamma_{1}, \cdots, \gamma_{n}\right\}$ be any finite subset of $\Gamma$ and define

$$
M_{\Gamma^{\prime}}=\left(\alpha_{i, j}^{\Gamma^{\prime}}\right)_{i, j=1}^{\infty}
$$

where

$$
\alpha_{i, j}^{r^{\prime}}= \begin{cases}F\left(\gamma_{\nu}\right) & \text { if } \lambda_{i}-\lambda_{j}=\gamma_{\nu} \in \Gamma^{\prime} \\ 0 & \text { otherwise }\end{cases}
$$

i.e., $M_{\Gamma^{\prime}} \sim\left(\Gamma, \Lambda, F_{\Gamma^{\prime}}\right)$ where $F_{\Gamma^{\prime}}(\gamma)=F(\gamma) I_{\Gamma^{\prime}}(\gamma)$. Since $F \geqq 0, \alpha_{i, j} \geqq$ $\alpha_{i, j}^{\Gamma^{\prime}} \geqq 0$ for $i, j \in N^{+}$, and Lemma 2 implies $|M|_{I} \geqq\left|M_{\Gamma^{\prime}}\right|_{I}$. But by Theorem 2

$$
\left|M_{\Gamma^{\prime}}\right|_{I}=\underset{x \in G}{\operatorname{ess} \sup }\left|\sum_{\gamma \in \Gamma^{\prime}}(\gamma, x) F_{\Gamma^{\prime}}(\gamma)\right|=\sum_{\gamma \in I^{\prime \prime}} F(\gamma)
$$

since $\sum_{\gamma \in \Gamma}(\gamma, x) F_{\Gamma^{\prime}}(\gamma)$ is continuous and $F_{\Gamma^{\prime}} \geqq 0$. This in turn implies

$$
|M|_{I} \geqq \sup _{\left|\Gamma^{\prime}\right|<+\infty} \sum_{\gamma \in I^{\prime}} F(\gamma)=+\infty .
$$

COROLlary 2. Under the hypothesis of Theorem 3.

$$
|M|=|M|_{I}=\sup _{i \in N^{+}}\left(\sum_{j \in N^{+}} \alpha_{i, j}\right)=\sup _{j \in N^{+}}\left(\sum_{i \in N^{+}} \alpha_{i, j}\right) .
$$

Proof. We prove only $|M|=|M|_{I}=\sup _{i \in N^{+}}\left(\sum_{j \in N^{+}} \alpha_{i, j}\right)$ the proof i of the other equality being similar. By Theorem 3. we need only verify $\sup _{i \in N^{+}}\left(\sum_{j \in N^{+}} \alpha_{i, j}\right)=\sum_{\gamma \in \Gamma} F(\gamma)$. First

$$
\sum_{j \in N^{+}} \alpha_{i, j}=\sum_{j \in N^{+}} F\left(\lambda_{i}-\lambda_{j}\right)=\sum_{\gamma \in \lambda_{2}-A} F(\gamma) \leqq \sum_{\gamma \in I} F(\gamma) .
$$


Let $\Gamma^{\prime}=\left\{\gamma_{1}, \cdots, \gamma_{n}\right\}$ be any finite subset of $\Gamma$ and let $\Gamma_{0}=$ $\left\{0,-\gamma_{1}, \cdots,-\gamma_{n}\right\}$. Condition (ii) insures the existence of an $\alpha \in \Gamma$ such that $\alpha+\Gamma_{0} \subseteq \Lambda$. In particular $\alpha \in \Lambda$, say $\alpha=\lambda_{k(\alpha)}$. But

$$
\Gamma^{\prime} \subseteq-\Gamma_{0}=\alpha-\left(\alpha+\Gamma_{0}\right) \subseteq \lambda_{k(\alpha)}-\Lambda,
$$

and thus for $i=k(\alpha)$ we have

$$
\sum_{j \in N^{+}} \alpha_{i, j}=\sum_{\gamma \in \lambda_{k(\alpha)}-\Lambda} F(\gamma) \geqq \sum_{\gamma \in \Gamma^{\prime}} F(\gamma)
$$

since $F \geqq 0$, and our assertion follows.

3. An application. In this section we apply the results of $\S 2$ to evaluate the norm of a special type of linear operator.

Definition 4. Let $T$ be the circle group, considered as the real numbers $R^{+} \bmod 2 \pi$, and let $L^{2}=L^{2}(T, d t)$ be the associated Hilbert function space with respect to normalized Lebesgue measure. Let $\mathscr{L l} \subseteq L^{2}$ be the submanifold

$$
\mathscr{L C}=\left\{f \in L^{2}: \int_{T} f(t) d t=0\right\} .
$$

Furthermore, let $\boldsymbol{Z}^{\prime}=\boldsymbol{Z} \sim\{0\}$ and for $a=\left\{a_{n}\right\}_{n \in Z^{\prime}} \in L^{1}\left(\boldsymbol{Z}^{\prime}\right)$ define $H_{a}: \mathscr{H C} \rightarrow \mathscr{L l}$ by

$$
\left(H_{a} f\right)(t)=\sum_{n \in Z^{\prime}} a_{n} f(n t)
$$

(where equality of functions is to be taken in the $L^{2}$ sense).

We now show that the mapping $a m H_{a}$ is a one-to-one bounded linear transformation from $L^{1}\left(\boldsymbol{Z}^{\prime}\right)$ into $\mathscr{C}^{*}$, the dual space of $\mathscr{C}$. For

$$
\begin{aligned}
& \left\|H_{a} f\right\|_{2}^{2}=\|\left.\sum_{n \in Z^{\prime}} a_{n} f(n t) \sum_{m \in Z^{\prime}} \overline{a_{m} f(m t)}\right|_{1} \\
= & \left\|\sum_{m, n \in Z^{\prime}} a_{n} \overline{a_{m}} f(n t) f \overline{(m t)}\right\|_{1} \leqq \sum_{m, n \in Z^{\prime}}\left|a_{n}\right|\left|a_{m}\right|\|f(n t) f(m t)\|_{1} \\
\leqq & \left\|\sum_{m, n \in Z^{\prime}}\left|a_{n}\right|\left|a_{m}\right|\right\| f(n t)\left\|_{2}\right\| f(m t)\left\|_{2}=\left(\sum_{n \in Z^{\prime}}\left|a_{n}\right|\right)^{2}\right\| f\left\|_{2}^{2}=\right\| a\left\|_{1}^{2}\right\| f \|_{2}^{2}
\end{aligned}
$$

since $\|f(n t)\|_{2}=\|f(t)\|_{2}$ for all $n \in Z^{\prime}$. Therefore $\left\|H_{a}\right\|_{o p} \leqq\|a\|_{1}$. Also, $f \in \mathscr{M}$ implies $H_{a} f \in \mathscr{M}$ since

$$
\int_{T}\left(H_{a} f\right)(t) d t=\sum_{n \in Z^{\prime}} a_{n} \int_{T} f(n t) d t=0 .
$$

Therefore, since $H_{a}$ is clearly linear, $H_{a} \in \mathscr{C}^{*}$ and the mapping 
$a m H_{a}$ is bounded and linear from $L^{1}\left(Z^{\prime}\right)$ to $\mathscr{C}^{*}$. Finally, the mapping is one-to-one since

$$
H_{a}\left(e^{i t}\right)=\sum_{n \in \boldsymbol{Z}^{\prime}} a_{n} e^{i n t}=0 \Leftrightarrow a=0 .
$$

We now apply Corollary 1 to evaluate the norm of $H_{a}$.

THEOREM 4. Let $a=\left\{a_{n}\right\}_{n \in Z^{\prime}} \in L^{1}\left(Z^{\prime}\right)$, and for $r \in Q^{\times}$let

$$
F(r)=\sum_{\substack{m / n=r \\ m, n \in Z^{\prime}}} a_{m} \bar{a}_{n}
$$

Then

$$
\left\|H_{a}\right\|_{o p}=\max _{x \in \widehat{Q}^{\times}}\left|\sum_{r \in Q^{\times}}(r, x) F(r)\right|^{\frac{1}{2}}
$$

where $\hat{Q}^{\times}$is the compact dual of the discrete group $Q^{\times}$.

Proof. Let $f \in \mathscr{H}$, and let the Fourier expansion of $f$ be

$$
f(t)=\sum_{m \in Z^{\prime}} b_{m} e^{i m t}
$$

Then

$$
\begin{aligned}
& \left(H_{a} f\right)(t)=\sum_{n \in \boldsymbol{Z}^{\prime}} a_{n} f(n t)=\sum_{n \in \boldsymbol{Z}^{\prime}} a_{n}\left[\sum_{m \in \boldsymbol{Z}^{\prime}} b_{m} e^{i m n t}\right] \\
= & \sum_{m, n \in \boldsymbol{Z}^{\prime}} a_{n} b_{m} e^{i m n t}=\sum_{p \in \boldsymbol{Z}^{\prime}} c_{p} e^{i p t},
\end{aligned}
$$

where $c_{p}=\sum_{m n=p m, n \in \boldsymbol{Z}^{\prime}} a_{n} b_{m}$, and $L^{2}$ convergence is the justification for the rearrangement of summation. Therefore

$$
\begin{gathered}
\left\|H_{a} f\right\|_{2}^{2}=\sum_{p \in \boldsymbol{Z}^{\prime}}\left|c_{p}\right|^{2}=\sum_{p \in \boldsymbol{Z}^{\prime}}\left(\sum_{\substack{m=p \\
m, n \in Z^{\prime}}} \overline{a_{n} b_{m}} \sum_{\substack{m^{\prime}, n^{\prime}=p \\
m^{\prime}, n^{\prime} \in \boldsymbol{Z}^{\prime}}} a_{n^{\prime}}, b_{m^{\prime}}\right) \\
=\sum_{\substack{m, n, m^{\prime}, n^{\prime} \in \boldsymbol{Z}^{\prime} \\
m n=m^{\prime}, n^{\prime}}} \bar{a}_{n} \bar{b}_{m} a_{n^{\prime}}, b_{m^{\prime}}=\sum_{m, m^{\prime} \in \boldsymbol{Z}^{\prime}}\left\{\left(\sum_{\substack{n, n^{\prime} \in \boldsymbol{Z}^{\prime} \\
n^{\prime} \mid n=m / m^{\prime}}} a_{n^{\prime}} \overline{a_{n}}\right) b_{m^{\prime}} \overline{b_{m^{\prime}}}\right\}
\end{gathered}
$$

where the manipulation of the quadruple sum is justified by absolute convergence:

$$
\begin{aligned}
& \sum_{\substack{m, n, m m^{\prime}, n^{\prime} \in \boldsymbol{Z}^{\prime} \\
m n=m^{\prime} n^{\prime}}}\left|\bar{a}_{n} \bar{b}_{m} a_{n^{\prime}}, b_{m^{\prime}}\right|=\sum_{n, n^{\prime} \in \boldsymbol{Z}^{\prime}}\left|a_{n}\right|\left|a_{n^{\prime}}\right|\left(\sum_{\substack{m, m^{\prime} \in \boldsymbol{Z}^{\prime} \\
n^{\prime} \mid n=m / m^{\prime}}}\left|b_{m}\right|\left(b_{m^{\prime}}\right)\right) \\
& \leqq \\
& \sum_{n, n^{\prime} \in \boldsymbol{Z}^{\prime}}\left|a_{n}\right|\left|a_{n^{\prime}}\right|\left(\sum_{m \in Z^{\prime}}\left|b_{m}\right|^{2}\right)=\|a\|_{1}^{2}\|f\|_{2}^{2}<+\infty
\end{aligned}
$$

by Cauchy-Schwarz. Upon setting 


$$
\alpha_{i, j}=\sum_{m / n=i / j} a_{m} \bar{a}_{n}=F\left(\frac{i}{j}\right) \quad \text { for } i, j \in Z^{\prime}
$$

and

$$
M=\left(\alpha_{i j}\right) \quad\left(\text { order } Z^{\prime}=(1,-1,2,-2, \cdots)\right)
$$

we obtain

$$
M \sim\left(Q^{\times}, Z^{\prime}, F\right)
$$

Also, upon identifying $\mathscr{C}$ with $\mathscr{L}^{2}$ by $f \leftrightarrow X_{f}=\left(b_{1}, b_{-1}, b_{2}, b_{-2}, \cdots\right)$ we have

$$
\left\|H_{a} f\right\|_{2}^{2}=\left(M X_{f}, X_{f}\right)
$$

But

$$
\begin{aligned}
\sum_{r \in Q^{\times}}|F(r)| & =\sum_{\substack{i, j \in \boldsymbol{Z}^{\prime} \\
(i, j)=1 \\
i>0}}\left|F\left(\frac{i}{j}\right)\right| \leqq \sum_{\substack{i, j \in \boldsymbol{Z}^{\prime} \\
(i, j)=1 \\
i>0}} \sum_{m / n=i / j}\left|a_{n}\right|\left|a_{m}\right| \\
& =\left(\sum_{n \in \boldsymbol{Z}^{\prime}}\left|a_{n}\right|\right)^{2}=\|a\|_{1}^{2}<+\infty,
\end{aligned}
$$

and hence

$$
f(x)=\sum_{r \in Q^{\times}}(r, x) F(r)
$$

is a continuous function on $\hat{Q}^{\times}$with Fourier transform $F$. The theorem follows upon applying Theorem 2 (Corollary 1 (i)) to $M \sim\left(Q, Z^{\prime}, F\right)$.

CoRollary 3. If $a=\left\{a_{n}\right\}_{n \in Z^{\prime}} \in L^{1}\left(Z^{\prime}\right)$ and $a_{n} \geqq 0$ for all $n \in Z^{\prime}$, then

$$
\left\|H_{a}\right\|_{o p}=\|a\|_{1}
$$

Proof. By Theorem 4,

$$
\left\|H_{a p}\right\|_{o p}=\max _{x \in \hat{Q}^{\times}}\left|\sum_{r \in Q^{\times}}(r, x) F(r)\right|^{\frac{1}{2}} \leqq\left(\sum_{r \in Q^{\times}}|F(r)|\right)^{\frac{1}{2}}=\|a\|_{1}
$$

since

$$
a_{n} \geqq 0,|F(r)|=\sum_{\substack{m / n=r \\ m, n \in Z^{\prime}}} a_{n} a_{m} \text {, and } \sum_{r \in Q^{\times}}|F(r)|=\|a\|_{1}^{2} \text {. }
$$

But upon setting $x=0$ we obtain 


$$
\left|\sum_{r \in Q^{\times}}(r, 0) F(r)\right|^{\frac{1}{2}}=\left|\sum_{r \in Q^{\times}} F(r)\right|^{\frac{1}{2}}=\left(\sum_{r \in Q^{\times}}|F(r)|\right)^{\frac{1}{2}}=\|a\|_{1},
$$

and thus the proof is complete.

\section{BIBLIOGRAPHY}

1. W. R. Emerson, Asymptotic results for certain sequences of integral operators defined over groups, J. Math. Mech. 17 (1968), 737-758.

2. - Ratio properties in locally compact groups, Trans. Amer. Math. Soc. 133 (1968), 179-204.

3. H. A. Krieger, Toeplitz operators on locally compact abelian groups, J. Math. Mech. 14 (1965), 439-478.

Received April 14, 1967, and in revised form November 22, 1968.

NEW YORK UNIVERSITY

NEW YORK, NEW YORK 


\section{PACIFIC JOURNAL OF MATHEMATICS}

\section{EDITORS}

H. ROYDEN

Stanford University

Stanford, California

\author{
R. R. Phelps \\ University of Washington \\ Seattle, Washington 98105
}

J. DugundJI

Department of Mathematics

University of Southern California

Los Angeles, California 90007

RICHARD ARENS

University of California

Los Angeles, California 90024

\section{ASSOCIATE EDITORS}

E. F. BECKenbaCH

B. H. NEUMANN

F. WOLF

K. YoSHIDA

\section{SUPPORTING INSTITUTIONS}

\author{
UNIVERSITY OF BRITISH COLUMBIA \\ CALIFORNIA INSTITUTE OF TECHNOLOGY \\ UNIVERSITY OF CALIFORNIA \\ MONTANA STATE UNIVERSITY \\ UNIVERSITY OF NEVADA \\ NEW MEXICO STATE UNIVERSITY \\ OREGON STATE UNIVERSITY \\ UNIVERSITY OF OREGON \\ OSAKA UNIVERSITY \\ UNIVERSITY OF SOUTHERN CALIFORNIA
}

\author{
STANFORD UNIVERSITY \\ UNIVERSITY OF TOKYO \\ UNIVERSITY OF UTAH \\ WASHINGTON STATE UNIVERSITY \\ UNIVERSITY OF WASHINGTON \\ $\stackrel{*}{*} \stackrel{*}{*} \stackrel{*}{*}$ AMERICAN MATHEMATICAL SOCIETY \\ CHEVRON RESEARCH CORPORATION \\ TRW SYSTEMS \\ NAVAL WEAPONS CENTER
}

The Supporting Institutions listed above contribute to the cost of publication of this Journal, but they are not owners or publishers and have no responsibility for its content or policies.

Mathematical papers intended for publication in the Pacific Journal of Mathematics should be in typed form or offset-reproduced, double spaced with large margins. Underline Greek letters in red, German in green, and script in blue. The first paragraph or two must be capable of being used separately as a synopsis of the entire paper. It should not contain references to the bibliography. Manuscripts, in duplicate if possible, may be sent to any one of the four editors. Please classify according to the scheme of Math. Rev. 36, 1539-1546. All other communications to the editors should be addressed to the managing editor, Richard Arens, University of California, Los Angeles, California, 90024.

50 reprints are provided free for each article; additional copies may be obtained at cost in multiples of 50 .

The Pacific Journal of Mathematics is published monthly. Effective with Volume 16 the price per volume (3 numbers) is $\$ 8.00$; single issues, $\$ 3.00$. Special price for current issues to individual faculty members of supporting institutions and to individual members of the American Mathematical Society: $\$ 4.00$ per volume; single issues $\$ 1.50$. Back numbers are available.

Subscriptions, orders for back numbers, and changes of address should be sent to Pacific Journal of Mathematics, 103 Highland Boulevard, Berkeley, California, 94708.

PUBLISHED BY PACIFIC JOURNAL OF MATHEMATICS, A NON-PROFIT CORPORATION

Printed at Kokusai Bunken Insatsusha (International Academic Printing Co., Ltd.), 7-17, Fujimi 2-chome, Chiyoda-ku, Tokyo, Japan. 


\section{Pacific Journal of Mathematics \\ Vol. 29, No. $3 \quad$ July, 1969}

Herbert James Alexander, Extending bounded holomorphic functions from certain subvarieties of a polydisc ...................... 485

Edward T. Cline, On an embedding property of generalized Carter

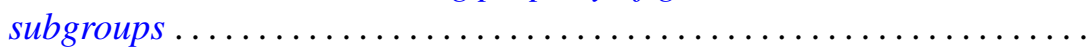

Roger Cuppens, On the decomposition of infinitely divisible characteristic functions with continuous Poisson spectrum. II ...............

William Richard Emerson, Translation kernels on discrete Abelian

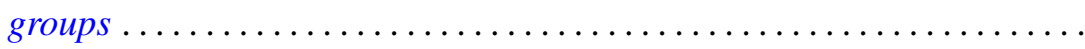

Robert William Gilmer, Jr., Power series rings over a Krull domain ....... 543

Julien O. Hennefeld, The Arens products and an imbedding theorem ...... 551

James Secord Howland, Embedded eigenvalues and virtual poles ........ 565

Bruce Ansgar Jensen, Infinite semigroups whose non-trivial homomorphs are all isomorphic .............................. 583

Michael Joseph Kascic, Jr., Polynomials in linear relations. II .......... 593

J. Gopala Krishna, Maximum term of a power series in one and several

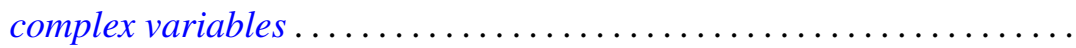

Renu Chakravarti Laskar, Eigenvalues of the adjacency matrix of cubic lattice graphs ...................................

Thomas Anthony Mc Cullough, Rational approximation on certain plane

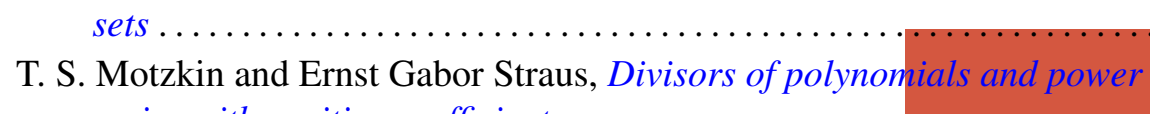
series with positive coefficients .

Graciano de Oliveira, Matrices with prescribed characteristic polynomial and a prescribed submatrix.

Graciano de Oliveira, Matrices with prescribed characteristic polynomial and a prescribed submatrix. II .

Donald Steven Passman, Exceptional 3/2-transitive permutation groups .................................

Grigorios Tsagas, A special deformation of the metric with no negative sectional curvature of a Riemannian space............

Joseph Zaks, Trivially extending decompositions of $E^{n}$ 